\title{
SIMULATION DE L'HABITAT PHYSIQUE DU BARBEAU FLUVIATILE (BARBUS BARBUS, L. 1758) : CHOIX DES MODĖLES BIOLOGIQUES ET SENSIBILITÉ DE LA RÉPONSE.
}

\author{
M. POUILLY, Y. SOUCHON
}

avec la collaboration technique de P. ROGER.

CEMAGREF, Division de Biologie des Ecosystèmes Aquatiques, Laboratoire d'Hydroécologie Quantitative, 3 bis Quai Chauveau, 69336 LYON Cedex 09, France.

\begin{abstract}
RÉSUMÉ
Des courbes monovariées et des modèles multivariés de préférence d'habitat du barbeau, Barbus barbus, ont été établis à partir de données récoltées sur 3 cours d'eau. Dans les deux cas (monovarié et multivarié), trois modèles locaux, correspondant aux données d'une rivière, et un modèle général, regroupant l'ensemble des données, ont été établis. La qualité de la prédiction et la sensibilité de la réponse lors de la simulation des capacités d'accueil d'un cours d'eau révèlent : 1) que les modèles multivariés ont une valeur prédictive plus forte que les courbes de préférence, 2) que les modèles construits à partir de jeux de données locaux sont plus performants que les modèles généraux, et 3) que la perte de précision est moindre dans le cas du modèle général multivarié.
\end{abstract}

Mots-clés : Préférences d'habitat, capacités d'accueil, modèles monovariés, modèles multivariés, Barbus barbus.

PHYSICAL HABITAT SIMULATION OF BARBEL (BARBUS BARBUS, L. 1758) : SELECTION OF BIOLOGICAL MODELS BASED ON PREDICTABILITY CRITERIA.

\begin{abstract}
Habitat suitability curves and habitat use factorial models were elaborated for Barbus barbus with data from 3 rivers. For the two cases, three local models, issued from data of one river, and one general model, constructed with all the data, were established. The predictive power and the sensitivity of the response showed that : 1) factorial models are better for prediction than monovariate models, 2 ) the local models were better than general models, and 3) the loss of predictive power was lower for the factorial general model than for the monovariate general model.
\end{abstract}

Key-words : Habitat use, carrying capacity, monovariate models, factorial models, Barbus barbus.

\section{INTRODUCTION}

La nécessité d'une meilleure prise en considération de la dimension physique des hydrosystèmes avant toute mesure de protection et de réhabilitation est désormais largement acceptée au niveau international (HUGHES et NOSS, 1992 ; OSBORNE et al., 1993). Ce constat s'est accompagné d'un développement de méthodes visant à apprécier la qualité de l'habitat à partir de déterminants agissant à différents niveaux spatiaux et temporels, depuis le bassin versant (hydrologie, macromorphologie) jusqu'à l'espace élémentaire directement perçu par les organismes et défini classiquement par des variables hydro- et morpho-dynamiques. 
Dans le cas des méthodes d'évaluation de la capacité d'accueil physique d'un cours d'eau, deux échelles sont classiquement distinguées :

- au niveau d'un tronçon de cours d'eau (MALAVOI, 1989) les variables telles que celles liées à la physico-chimie ou à la ressource trophique définissent un «hydroclimat" considéré comme homogène. II s'agit d'un premier niveau de hiérarchisation qui détermine la distribution des populations, au sein de l'aire géographique de répartition des espèces, et un niveau de productivité potentielle.

- au niveau du microhabitat (ALLEE et al., 1949 ; BOVEE, 1982), les variables hydro- et morpho-dynamiques locales telles que le substrat, la vitesse du courant et la hauteur d'eau ou la végétation apparaissent déterminantes. C'est un niveau d'étude pertinent pour décrire la sélection de l'habitat par les poissons (GORMAN et KARR, 1978 ; SCHLOSSER, 1982 ; MOYLE et VONDRACEK, 1985). Plusieurs modèles biologiques d'utilisation et de préférence vis-à-vis de l'habitat ont été proposés en respectant cette logique. Ils permettent l'estimation des capacités d'accueil physiques des cours d'eau, grâce à un couplage avec des modèles hydrauliques selon une démarche de type méthode des microhabitats (BOVEE, 1982 ; ORTH 1987 ; MALAVOI et SOUCHON, 1989).

Les modèles biologiques les plus largement répandus se présentent sous la forme de courbes de préférence monovariées pour un paramètre physique. Ils sont établis par synthèse bibliographique, enquêtes auprès d'experts ou mesures directes (BOVEE, 1982 ; HEGGENES et al., 1990). Certains auteurs ont proposé des courbes bivariées (HANSON, 1988 ; VOOS et LIFTON, 1988). Ces modèles ont fait l'objet de critiques (MATHUR et al., 1985 ; ORTH, 1987 ; SHIRVELL, 1989 ; SOUCHON et al., 1989), notamment :

- l'hypothèse d'indépendance des paramètres physiques telle que le suggèrent BOVEE et MILHOUS (1978) ne se vérifie pas notamment entre la hauteur d'eau et la vitesse du courant (ORTH et MAUGHAN, 1982).

- la dynamique saisonnière de l'utilisation de l'habitat par les poissons peut être reliée à une variation de la disponibilité en habitat (GROSSMAN et al., 1987) qui n'est généralement pas prise en compte.

Le recours à des modèles biologiques bâtis à partir de statistiques multivariées (SOUCHON et al., 1989 ; THOMAS et BOVEE, 1993) permet de s'affranchir de ces critiques.

La plupart des modèles ont été développés pour des espèces économiquement intéressantes comme les Salmonidés. Des progrès restent à faire pour valider ces modèles dans les cours d'eau européens et en développer de nouveaux pour des espèces caractéristiques des peuplements des rivières de moyenne envergure (ordre 5 à 7 ). Les espèces de poissons colonisant ces milieux présentent des exigences écologiques et des stratégies d'occupation de l'habitat complexes (BARAS et CHERRY, 1990 pour le barbeau).

Dans ce contexte de développement el d'adaptation de la méthode des microhabitats pour son utilisation dans des cours d'eau de moyenne envergure, nous présentons les bases méthodologiques de mise au point de modèles biologiques multivariés. A partir d'un exemple sur le barbeau fluviatile (Barbus barbus, L.1758), espèce de référence de la zone à barbeau (HUET, 1949), nous avons construit deux types de modèles biologiques : des modèles monovariés de type courbes de préférence et des modèles multivariés réalisés à partir d'un couplage des caractéristiques des habitats et de leur contenu biologique. Les objectifs de cet article sont :

- la détermination de la valeur prédictive en comparant pour les deux types de modèles, un modèle général (établi à partir d'un jeu de données provenant de trois rivières) et des modèles locaux (relatifs à une rivière).

- le couplage de ces différents modèles biologiques avec une simulation physique d'une portion du Rhône court-circuité de Rochemaure, selon la procédure décrite par BOVEE (1982) et redéfinie par SOUCHON et al. (1989) et MALAVOI et SOUCHON (1989), ceci pour analyser les tendances observées en termes de variations d'habitat potentiel en fonction des débits. 


\section{MATÉRIEL ET MÉTHODE}

\section{Echantillonnage}

Un échantillonnage stratifié, en fonction des faciès d'écoulement (MALAVOI, 1989) et de la position en berge ou dans le chenal, est réalisé sur un tronçon de rivière (MALAVOI, 1989) de façon à décrire l'ensemble des habitats disponibles. Dans chaque strate, des zones de pêches dénommées ambiances (MALAVOI, 1989 ; POUILLY, 1994), sont choisies aléatoirement. La taille des ambiances est fonction de la taille de la strate sur laquelle elle se situe, $95 \%$ des ambiances ont une taille comprise entre 5 et $50 \mathrm{~m}^{2}$ (POUILLY, 1994).

La capture des poissons se fait par pêche électrique (boîtier de type «Heron» délivrant un courant continu redressé d'intensité de 3 à $5 \mathrm{~A}$. pour un voltage de 200 à $600 \mathrm{~V}$.). Tous les poissons sont déterminés à l'espèce puis mesurés (longueur totale $\pm 1 \mathrm{~mm}$ ). Les pêches sont réalisées uniquement pendant le jour.

Les conditions physiques des ambiances sont caractérisées à partir des paramètres : hauteur d'eau (HT), vitesse moyenne du courant sur la colonne d'eau (VT, mesurée à l'aide d'un micromoulinet de type OTT/C31), et diamètre du substrat le plus grossier (SGR). La distinction essentielle entre l'échantillonnage classique par point focal ou EPA (NELVA et al., 1979) et l'ambiance réside dans la mesure des variables sur 4 à 12 points ( 6 en moyenne) de manière à représenter la diversité des conditions physiques élémentaires constituant un habitat.

Au cours de 14 campagnes de pêche (réalisées entre mars 1989 et octobre 1991) 668 ambiances ont été décrites sur des tronçons de la zone à barbeau de 3 cours d'eau du bassin rhodanien : I'Ain (ordre 6, pente $1.2 \mathrm{~m}^{\mathrm{km}} \mathrm{km}^{-1}$, module $125 \mathrm{~m}^{3} . \mathrm{s}^{-1}$, largeur $90 \mathrm{~m}$ ), l'Ardèche (ordre 7 , pente $3.4 \mathrm{~m} . \mathrm{km}^{-1}$, module $29 \mathrm{~m}^{3} . \mathrm{s}^{-1}$, largeur $50 \mathrm{~m}$ ) et la portion courtcircuitée du Rhône à Montélimar (ordre 9 , pente $0.9 \mathrm{~m} . \mathrm{km}^{-1}$, module (débit réservé) $15 \mathrm{~m}^{3} . \mathrm{s}^{-1}$, largeur $80 \mathrm{~m}$ ).

\section{Analyses descriptives}

\section{Courbes de préférence}

Les courbes de préférence pour la hauteur d'eau, la vitesse du courant et le substrat sont déterminées à partir des valeurs moyennes prises par ces variables dans les ambiances. Les variables physiques ont été découpées en classes les plus restreintes possibles. A chaque classe est associé un coefficient de préférence, pondéré par la disponibilité en habitat, calculé par la formule :

$$
\mathrm{C}_{(\mathrm{Xi})}=\frac{\text { (nombre d'individus associés à la classe } \mathrm{i} / \text { nombre total d'individus) }}{\text { (nombre de mesures dans la classe } \mathrm{i} / \text { nombre total de mesures) }}
$$

Les courbes sont normalisées à 1 .

\section{Modèles multivariés}

Les seconds modèles sont générés à partir d'une analyse multivariée des données. Pour analyser simultanément les tableaux de milieu et de faune, nous utilisons la méthode de couplage décrite par CAZES (1980) et généralisée par CHESSEL et DOLEDEC (1991). Cette démarche consiste en l'analyse du tableau de contingence croisant la présence ou l'absence de chaque espèce avec les classes des variables physiques (exprimées en pourcentage de représentation par ambiance). Pour optimiser l'analyse, les variables ont regroupées en 6 classes pour la hauteur d'eau (<20; ]20-30] ; ]30-40] ; 140-60] ; ]60-90] ; $>90 \mathrm{~cm}) ; 7$ classes pour la vitesse du courant $(0 ;] 0-5]$; ]5-15] ; ]15-40] ; ]40-50] ; ]50-90] ; $\left.>90 \mathrm{~cm} . \mathrm{s}^{-1}\right)$; et 6 classes pour le diamètre du substrat $\left.(<2 ;] 2-32\right]$; ]32-64] ; ]64-128] ; 1128-256] ; > 256mm). L'Analyse Factorielle des Correspondances (AFC) de ce tableau permet de déduire les relations qui existent entre les deux groupes de variables (MERCIER et al., 1992 ; DOLEDEC et CHESSEL, 1994). L'intérêt du couplage tient au fait que les cartes factorielles obtenues rendront compte de la localisation des espèces dans les 
habitats tout en conservant l'information sur les associations biologiques contenues dans le tableau de captures. L'espace factoriel issu de cette analyse conduit à une typologie des habitats, outil à partir duquel on peut mettre en oeuvre des procédures prédictives.

\section{Analyses prédictives}

Les prédictions ne sont vérifiées que pour les ambiances où l'espèce a été capturée. La présence d'une espèce dans une ambiance indique un habitat sélectionné par l'animal ; mais l'absence d'une espèce n'implique pas que l'ambiance lui soit défavorable. Tous les espaces favorables ne sont pas colonisés simultanément : les poissons ont des habitats différents selon l'activité qu'ils réalisent (repos et alimentation) à différentes périodes de la journée (BARAS et CHERRY, 1990).

\section{A partir des courbes de préférence monovariées}

Pour chaque point de mesure décrivant une ambiance, une valeur d'habitat (VH) est calculée en multipliant les coefficients de préférence normalisés $\left(C_{(X i)}\right)$ des trois variables comme dans la méthode des microhabitats:

$$
\mathrm{VH}_{\mathrm{i}}=\mathrm{C}_{(\mathrm{VTi})} \times \mathrm{C}_{(\mathrm{HTi})} \times \mathrm{C}_{(\mathrm{SGRi})}
$$

La valeur d'habitat globale de l'ambiance est la moyenne des valeurs d'habitat calculées pour chaque point de mesure.

Deux histogrammes des fréquences de valeurs d'habitat sont tracés, l'un correspondant aux ambiances où l'espèce a été capturée, l'autre aux ambiances où l'espèce est absente. La prédiction est empiriquement réalisée par affectation barycentrique par rapport à la médiane de l'un des deux histogrammes présence ou absence.

\section{A partir du couplage multivarié}

Il est possible d'effectuer une analyse discriminante à partir des coordonnées factorielles de l'AFC. Cette analyse discriminante classe les ambiances en mode favorable ou défavorable à partir de la présence ou de l'absence des espèces.

\section{Qualité prédictive des modèles}

Une procédure de tests croisés est réalisée : chaque ambiance constitue tour à tour un échantillon test par rapport au modèle construit à partir des $\mathrm{N}-1$ ambiances de l'échantillon total. Les valeurs prédites et observées sont comparées pour cet échantillon test. $\mathrm{N}$ itérations permettent d'obtenir un pourcentage de bonne prédiction qui représente une mesure de la qualité de la prédiction. Ce type de procédure permet de conserver un jeu de données test indépendant du jeu de données ayant servi à l'élaboration des modèles.

\section{Simulation de la capacité d'accueil physique}

Les capacités d'accueil physiques potentielles ont été simulées pour différents débits en appliquant la méthode des microhabitats (BOVEE, 1982 ; MALAVOI et SOUCHON, 1989) sur une station du Rhône court-circuité de Montélimar (POUILLY et SOUCHON, 1989), servant d'exemple. La méthode des microhabitats consiste à coupler un modèle hydraulique et un modèle biologique. Un modèle hydraulique monodimensionnel avec redistribution latérale des vitesses, (BAUME et POIRSON, 1984 ; TROCHERIE, 1987) permet de recalculer, pour différents débits, les valeurs de hauteur d'eau, vitesse du courant et substrat pour chacun des habitats élémentaires décrits systématiquement lors d'une campagne de terrain. Le modèle biologique donne la qualité de ces habitats élémentaires vis-à-vis du taxon étudié (les deux types de modèles biologiques décrits plus haut ont été successivement employés). Les résultats expriment alors une surface potentiellement utilisable par le taxon. L'évolution de ces surfaces en fonction du débit constitue une aide pour la détermination des débits biologiques à réserver dans des études de cas de gestion des rivières. Les simulations ont été réalisées en utilisant le logiciel EV̈HA développé au CEMAGREF de Lyon (GINOT et SOUCHON, 1992). 


\section{RÉSULTATS}

Au total, 15031 poissons appartenant à 30 espèces ont été capturés dans les 668 ambiances. Sur cet ensemble, les espèces représentant moins de $1 \%$ de l'effectif total ont été éliminées de l'analyse. Les autres espèces ont été réparties en classes de taille d'après les ruptures dans les histogrammes de fréquence des longueurs.

Les modèles monovariés et multivariés ont été établis pour les adultes de barbeau (> $220 \mathrm{~mm}$ de longueur totale). Cette classe de taille représente $3,9 \%$ de l'ensemble du peuplement soit 585 individus capturés sur 98 ambiances.

\section{Courbes de préférence pour le Barbeau}

Les courbes de préférence pour les variables hauteur d'eau et vitesse moyenne du courant montrent des maxima pour des hauteur d'eau de l'ordre de 60 puis de $200 \mathrm{~cm}$ et pour des vitesses de courant de l'ordre de 7.5 puis $60 \mathrm{~cm} . \mathrm{s}^{-1}$ (fig.1). Une préférence apparaît pour le substrat constitué d'éléments de grande taille.

\section{Analyse multivariée}

L'analyse multivariée $(79,3 \%$ de variabilité expliquée sur les trois premiers axes ; seuls les deux premiers axes ont été représentés en figure 2) fait ressortir l'organisation globale du peuplement par rapport aux trois variables hauteur d'eau, vitesse du courant et substrat. Le premier axe exprime un gradient positif de vitesse de courant et le second un gradient négatif de hauteur d'eau (fig.2a). Au niveau biologique, les espèces s'organisent d'une part en fonction de leur rhéophilie (sur le premier axe) et d'autre part en fonction de leur taille (sur le second axe) (fig.2b). Dans cet ensemble le barbeau adulte se trouve associé aux hauteurs d'eau intermédiaires (classes HT3 et HT4) et aux fortes vitesses de courant. La répartition des ambiances où des barbeaux adultes ont été capturés par rapport à l'ensemble des ambiances prospectées illustre cette préférence pour les habitats lotiques et relativement peu profonds (fig.2c).

\section{Comparaison de la qualité de prédiction des modèles}

Le tableau 1 résume les estimations des capacités prédictives des modèles (pourcentage d'ambiances où les modèles prédisent la présence du barbeau adulte et sur lesquelles ce taxon a été capturé). La qualité de prédiction des modèles monovariés et multivariés apparaît relativement comparable (> $65 \%$ ) lorsqu'ils sont appliqués sur les jeux de données dont ils sont issus (modèles "locaux", correspondant à un cours d'eau, testés "localement"). Par contre l'utilisation d'un modèle général, regroupant l'ensemble des données, sur des données locales montre dans le cas du modèle monovarié une perte de précision importante (jusqu'à $40 \%$ ) alors qu'elle est relativement faible dans le cas du modèle multivarié (jusqu'à 12,8\%).

\section{modèles}

Sensibilité de l'évolution des capacités d'accueil physique en fonction des

La figure 3 donne l'évolution des surfaces potentiellement utilisables (simulées par la méthode des microhabitats) pour chacun des modèles "locaux" et "généraux", monovariés et multivariés (les valeurs de surface utilisable ne sont pas comparables entre les deux types de modèles en raison de modes de calcul non similaires). Les modèles monovariés se traduisent par une évolution monotone et peu sensible à l'élévation du débit (fig.3a). De plus, il y a des différences dans les valeurs de surface utilisable entre les modèles : les modèles construits à partir d'un plus grand nombre de données déterminent des surfaces utilisables plus importantes. Ceci est la résultante de l'augmentation de la gamme de conditions favorables lorsque les données sont plus nombreuses ou proviennent de cours d'eau où les individus ont des préferendums décalés.

L'évolution produite par les simulations effectuées à partir des modèles multivariés, montre une augmentation des surfaces utilisables qui est tout d'abord rapide puis se ralentit ensuite lorsque le débit augmente (fig.3b). Contrairement aux courbes de préférence monovariées, les modèles multivariés engendrent sensiblement la même réponse quelle que soit leur origine. Ceci à l'exception du modèle provenant de la rivière Ain dont le modèle général prédit correctement seulement $51,7 \%$ des données, contre 83,3 et $85,5 \%$ pour les autres cours d'eau. 

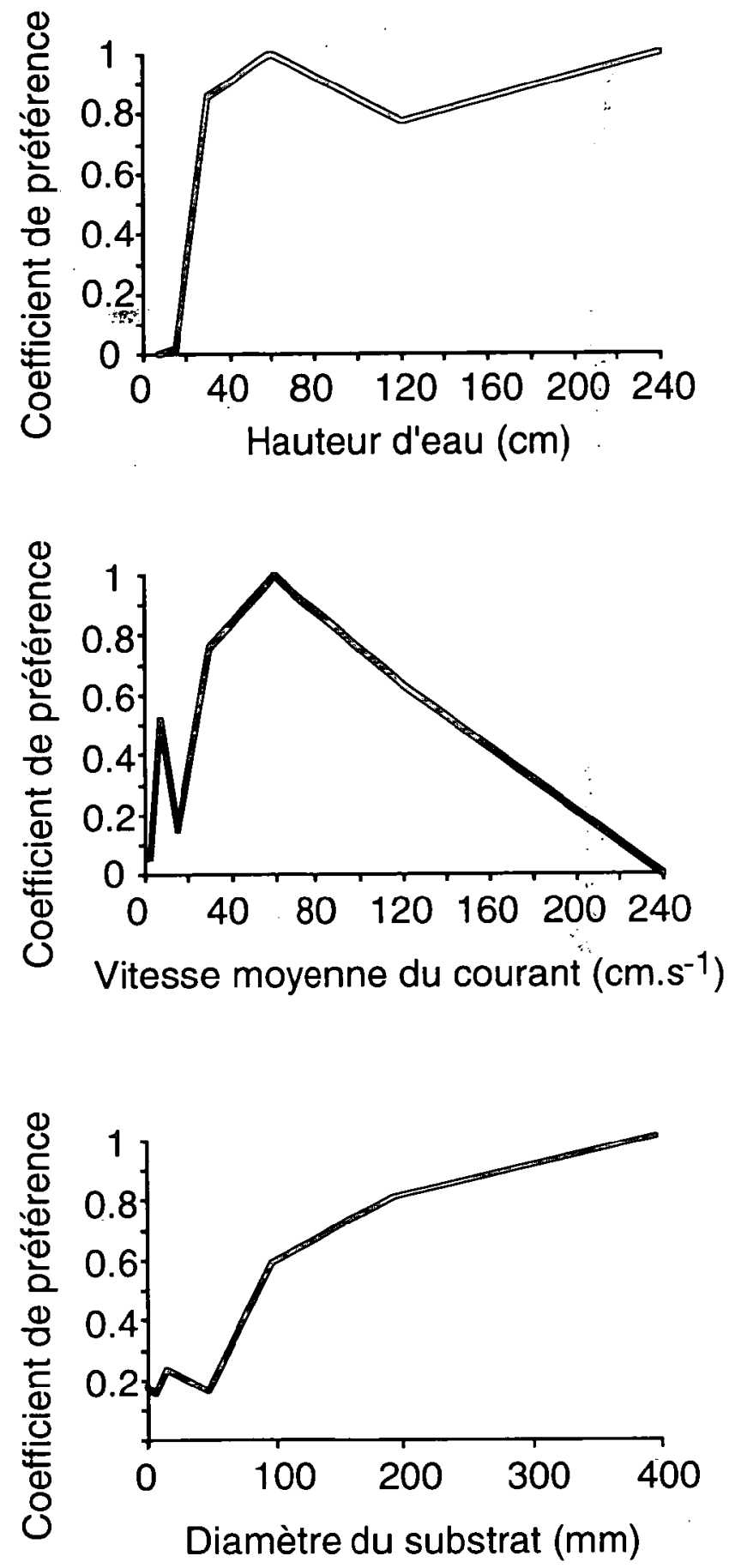

Figure 1 : Courbes de préférence du barbeau (Barbus barbus) de taille supérieure à $220 \mathrm{~mm}$, pour les variables hauteur d'eau, vitesse moyenne du courant sur la colonne d'eau et diamètre du substrat, établies à partir de 585 individus répartis dans 668 ambiances prospectées sur l'Ain, l'Ardèche et le Rhône.

Figure 1 : Preference curves of barbel (Barbus barbus) greater than $220 \mathrm{~mm}$ of total length, for water depth, mean current velocity and substrate diameter, established with 585 fishes captured in 668 «ambiances» from rivers Ain, Ardèche and Rhône. 

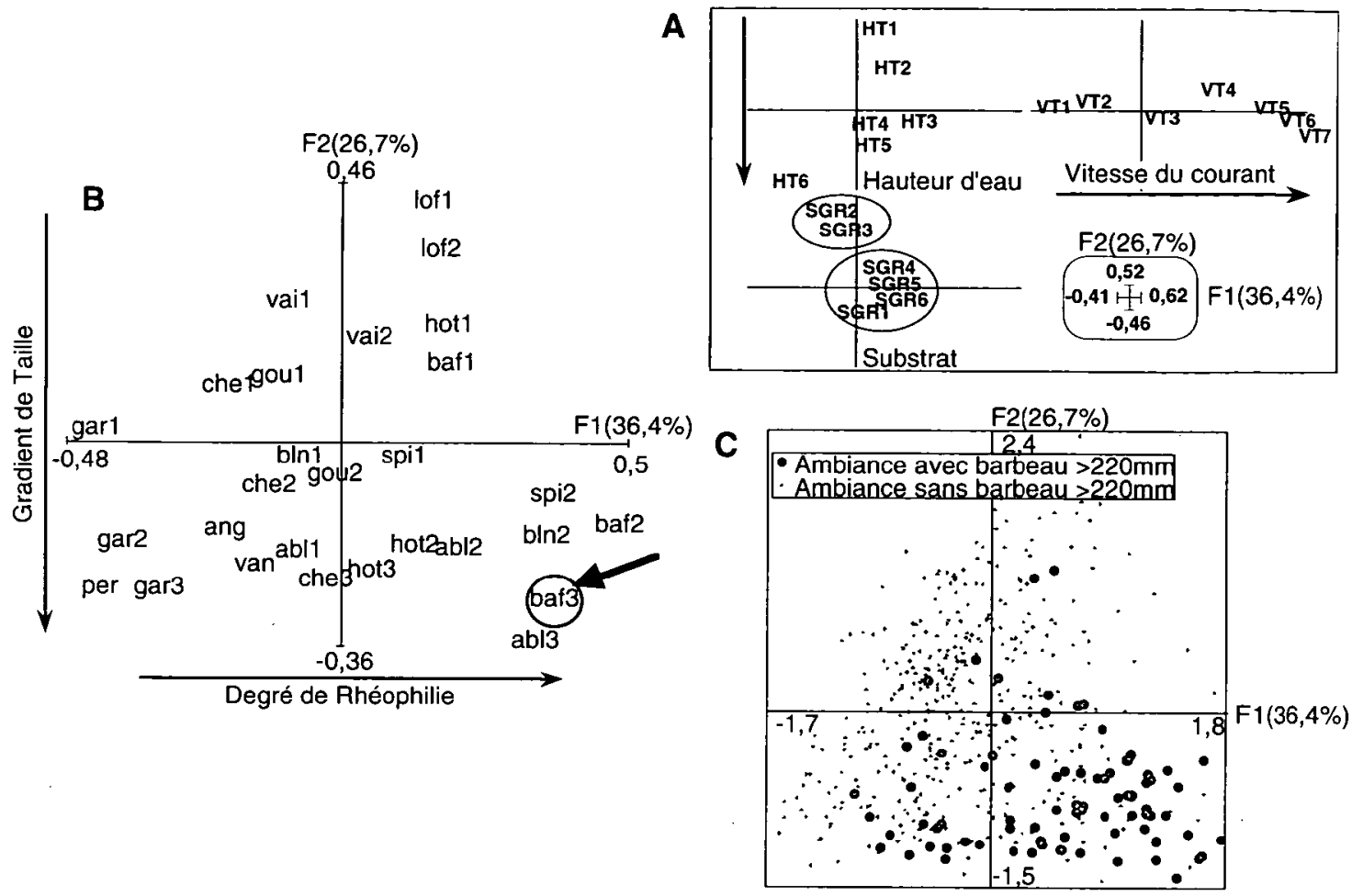

Figure 2 : Analyse Factorielle des Correspondances du tableau couplant la présence des espèces et les variables physiques.

A- Position moyenne des modalités physiques sur le plan F1F2.

Hauteur d'eau (water depth) : HT1 $=<20 \mathrm{~cm} ; \mathrm{HT2}=20-30 \mathrm{~cm} ; \mathrm{HT} 3=30-40 \mathrm{~cm}$; HT4 $=40-60 \mathrm{~cm} ; \mathrm{HT5}=60-90 \mathrm{~cm} ; \mathrm{HT6}=>90 \mathrm{~cm}$.

Vitesse du courant (current velocity) : VT1 $=0 \mathrm{~cm} / \mathrm{s} ; \mathrm{VT2}=0-5 \mathrm{~cm} / \mathrm{s} ; \mathrm{VT} 3=$ $5-15 \mathrm{~cm} / \mathrm{s} ;$ VT4 $=15-40 \mathrm{~cm} / \mathrm{s} ;$ VT5 $=40-50 \mathrm{~cm} / \mathrm{s} ;$ VT6 $=50-90 \mathrm{~cm} / \mathrm{s} ; \mathrm{VT7}=$ $>90 \mathrm{~cm} / \mathrm{s}$.

Diamètre du substrat (substrate diameter) : SG1 $=<2 \mathrm{~mm} ; \mathrm{SG2}=2-32 \mathrm{~mm}$; $S G 3=32-64 \mathrm{~mm} ; \mathrm{SG} 4=64-128 \mathrm{~mm} ; \mathrm{SG} 5=128-256 \mathrm{~mm} ; \mathrm{SG} 6=>256 \mathrm{~mm}$.

B- Position moyenne des espèces sur le plan F1F2.

$A B L 1=$ ablette $<80 \mathrm{~mm} ; A B L 2=$ ablette $80-120 \mathrm{~mm} ; A B L 3=$ ablette $>120 \mathrm{~mm}$; ANG = anguille ; BAF1 = barbeau $<90 \mathrm{~mm}$; BAF2 = barbeau $90-220 \mathrm{~mm}$; BAF3 = barbeau $>220 \mathrm{~mm} ;$ BLN1 = blageon $<80 \mathrm{~mm} ;$ BLN2 = blageon $>80 \mathrm{~mm}$; $\mathrm{CHE1}=$ chevaine $<80 \mathrm{~mm}$; CHE2 = chevaine $80-170 \mathrm{~mm}$; $\mathrm{CHE3}=$ chevaine $>170 \mathrm{~mm}$; GAR1 = gardon $<60 \mathrm{~mm}$; GAR2 = gardon $60-110 \mathrm{~mm}$; GAR3 = gardon $>110 \mathrm{~mm} ;$ GOU1 = goujon $<70 \mathrm{~mm} ;$ GOU2 $=$ goujon $>70 \mathrm{~mm}$; HOT1 = hotu $<80 \mathrm{~mm} ;$ HOT2 = hotu $80-190 \mathrm{~mm} ;$ HOT3 $=$ hotu $>190 \mathrm{~mm}$; LOF1 $=$ loche franche $<60 \mathrm{~mm}$; LOF2 $=$ loche franche $>60 \mathrm{~mm} ;$ PER $=$ perche fluviatile ; SPI1 = spirlin $<70 \mathrm{~mm}$; SPI2 $=$ spirlin $>70 \mathrm{~mm}$; VAl1= vairon $<40 \mathrm{~mm} ; \mathrm{VAl} 2$ = vairon $>40 \mathrm{~mm} ; \mathrm{VAN}=$ vandoise

C- Position des ambiances sur le premier plan factoriel. Les ambiances où il y a eu captures de barbeau adulte (Barbus barbus $>220 \mathrm{~mm}$ ) sont différenciées par les cercles grisés.

Figure 2 : Correspondence analysis of the table combining physical and biological informations.

A- Mean scores of physical categories on the two first axes.

B- Mean scores of species on the two first axes.

C- Position of "ambiances" on the two first axes. "Ambiances" where adult barbel (Barbus barbus $\mathbf{7 2 2 0} \mathrm{mm}$ ) was captured are distinguished by grey circles. 
Tableau I: Pourcentages de bonnes prédictions des courbes monovariées et des modèles multivariés.

Table I : Percentages of right predictions for factorial and multivariate models.

\begin{tabular}{lcccc}
\hline & \multirow{2}{*}{ Modèle général } & \multicolumn{3}{c}{ Modèles locaux } \\
\cline { 4 - 5 } & & Ain & Ardèche & Rhône \\
\hline courbes monovariées : & & & & \\
Modèle génèral & $77,50 \%$ & $32,30 \%$ & $54,50 \%$ & $83,30 \%$ \\
Modèles locaux & & $71,00 \%$ & $90,90 \%$ & $83,30 \%$ \\
$\begin{array}{l}\text { modèles multivariés : } \\
\text { Modèle général }\end{array}$ & $82,70 \%$ & $51,70 \%$ & $85,50 \%$ & $83,30 \%$ \\
$\begin{array}{l}\text { Modèles locaux } \\
\text { nombre d'ambiances }\end{array}$ & 668 & $64,50 \%$ & $85,50 \%$ & $75,00 \%$ \\
nombre d'ambiances & & 223 & 288 & 157 \\
avec du barbeau adulte & 98 & 31 & 55 & 12 \\
nombre d'individus & 585 & 91 & 433 & 61 \\
\hline
\end{tabular}

\section{DISCUSSION}

La nécessité de quantification des capacités d'accueil des cours d'eau dans une optique de gestion est à l'origine du développement récent des modèles d'habitat. Chez les poissons, plusieurs facteurs influencent le choix du microhabitat et par là même la construction des modèles : l'espèce, le stade de développement, la saison, le type de cours d'eau, la disponibilité en nourriture et les relations de voisinage (compétition, prédation). L'approche monovariée fournit une résolution restreinte des dynamiques spatiale et temporelle qui résultent de l'influence de ces facteurs : seule la multiplication du nombre de courbes peut permettre d'envisager une amélioration des procédures prédictives. Ceci conduit à préconiser l'emploi de courbes ou de modèles pour chaque site. Pour des raisons évidentes de coût et de temps de mise en oeuvre, il est primordial de chercher à connaître la qualité du pouvoir prédictif de modèles généraux. En prenant le barbeau comme exemple, nous avons testé les propriétés prédictives de modèles locaux et de modèles généraux construits par approches monovariées ou multivariées.

Les résultats obtenus sont à rapprocher des études plus éthologiques ménées sur le comportement du barbeau (PELZ et KASTLE 1989, BARAS et CHERRY 1990, BARAS 1992, BARAS 1993). Ces études, en démontrant une utilisation crépusculaire et nocturne des habitats d'alimentation et une utilisation diurne d'habitat de repos, montrent la limite de données issues d'échantillonnage de jour. Cependant, dans le cas de la hauteur d'eau et de la vitesse du courant, les courbes de préférence établies sont bimodales et chacun des pics de préférence pourraient correspondre à ces deux types d'habitat. BARAS (1992), dans une étude par radiotélémetrie, trouve pour ces deux habitats des maxima de l'ordre de 20 et $80 \mathrm{~cm}$ pour les hauteurs d'eau et de 5 et $35 \mathrm{~cm} . \mathrm{s}^{-1}$ pour des vitesses de courant. Ces valeurs sont proches de celles issues de notre jeu de données pour la vitesse du courant, et plus faibles pour la hauteur d'eau. Ce déplacement des pics de préférence peut s'expliquer par la dimension des systèmes échantillonnés. En effet, le secteur de l'Ourthe étudié par Baras (1992) est plus petit que les secteurs que nous avons prospectés (largeur $26.5 \mathrm{~m}$, module $\left.21 \mathrm{~m}^{3} . \mathrm{s}^{-1}\right)$. Les hauteurs d'eau doivent donc y être plus faibles, et parallèlement les pics de préférence des Barbeaux pour la hauteur d'eau apparaissent plus faibles. 

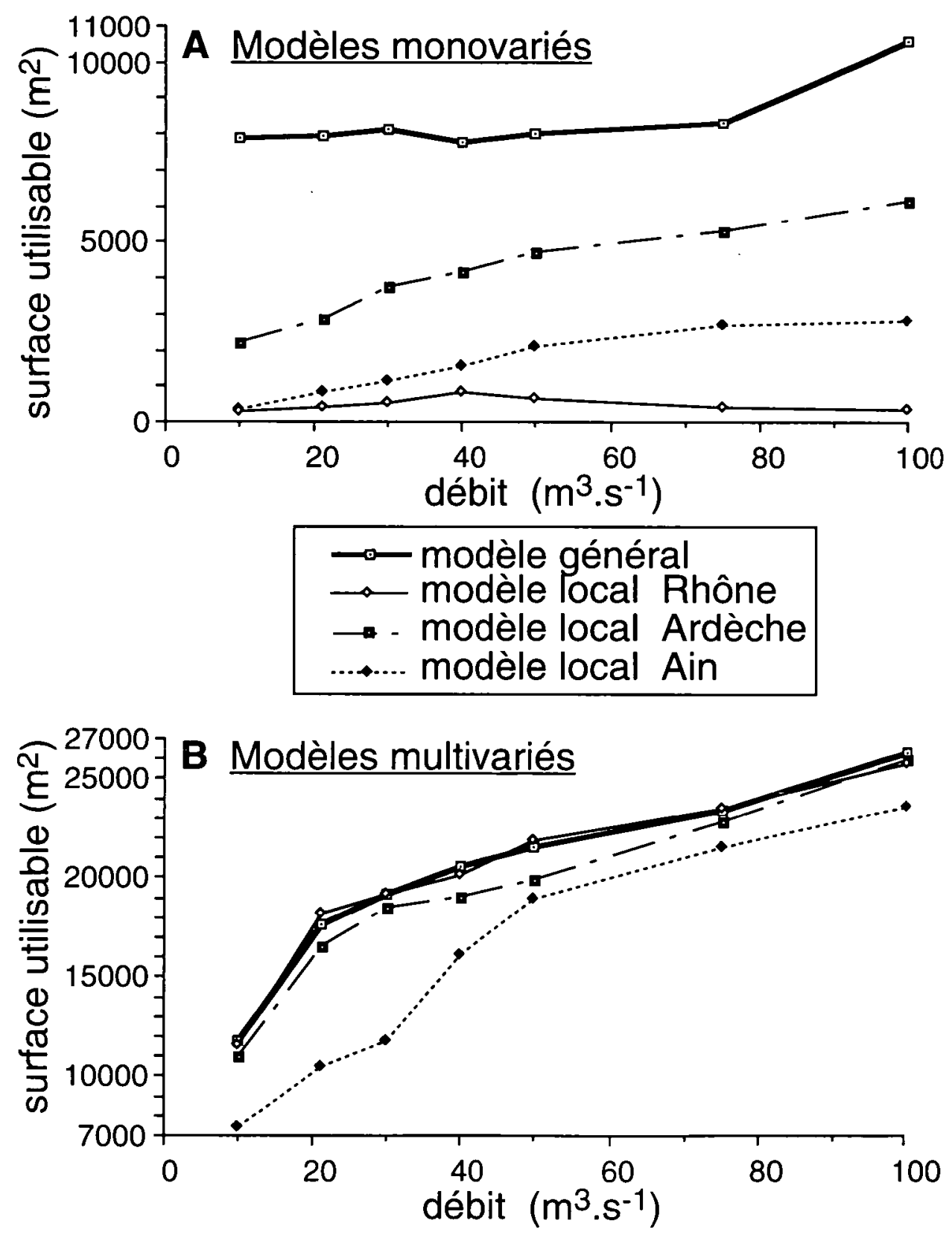

Figure 3 : Evolution en fonction du débit de la surface utilisable par le barbeau (Barbus barbus) sur une station du Rhône court-circuité de Montélimar (France). A. calculée à partir des courbes de préférence monovariées. B. calculée à partir des modèles multivariés.

Figure 3 : Evolution of the usable area for the barbel (Barbus barbus) in a station of the Rhône River near Montélimar (France). A. calculated with monovariate preference curves. B. calculated with factorial models. 
Les simulations ont été réalisées jusqu'au débit de $100 \mathrm{~m}^{3} \cdot \mathrm{s}^{-1}$, limite imposée par le domaine de validité du modèle hydraulique, alors que le débit morphogène du bras courtcircuité est de l'ordre de $1400 \mathrm{~m}^{3} . \mathrm{s}^{-1}$ (module du Rhône au niveau de Montélimar). Pendant les fortes eaux, le comportement des poissons est modifié : les barbeaux cherchent des conditions de refuge hydraulique (BARAS, 1993) qui ne correspondent plus aux mêmes préférences. Ces difficultés de prise en compte de toute la dynamique spatio-temporelle de l'habitat affectent les interprétations de la méthode des microhabitats et les limitent à l'analyse des débits faibles et moyens. D'où un domaine d'application privilégié de la méthode en tant qu'outil d'aide à la détermination des débits réservés.

La prise en compte, dans les modèles biologiques, des particularités spatiotemporelles de l'utilisation de l'habitat à une échelle très fine (infra-journalière), ou à des échelles plus larges pour intégrer les comportements de reproduction ou de refuge en cas de crues par exemple, reste un objectif à réaliser pour obtenir une image plus dynamique des potentialités d'accueil d'une rivière.

Toutefois, le choix de modèles multivariés généraux apparaît justifié dans les applications s'inscrivant dans une logique de gestion des cours d'eau. L'exemple présenté ici illustre la pertinence des modèles multivariés qui produisent des réponses homogènes quelle que soit leur origine, et proches des comportements constatés dans les zones salmonicoles (voir BOVEE, 1982 ; LEONARD et al., 1986 ; SOUCHON et al., 1989). Les modèles monovariés, quant à eux, produisent des réponses différentes et moins sensibles à l'évolution des caractéristiques hydrauliques dans la gamme des débits testés. Le choix du modèle influence donc l'estimation de la capacité d'accueil potentielle et doit donc être correctement raisonné lors de la mise en oeuvre de la méthode des microhabitats. Ceci rend compte de l'importance d'une description précise de toutes les étapes intermédiaires de l'analyse (choix des stations, méthode de mesure et de modélisation hydraulique, choix du modèle biologique utilisé).Dans la gamme modélisée, l'interprétation des évolutions des capacités d'accueil reste délicate dans le cas des modèles monovariés. Pour les modèles multivariés, on peut indiquer qu'un débit inférieur à $40 \mathrm{~m}^{3} \cdot \mathrm{s}^{-1}$ correspond à une perte très nette de la capacité d'accueil pour la station étudiée.

Le modèle multiplicatif des microhabitats utilisant des courbes de préférence monovariées suppose que les poissons sélectionnent leurs habitats en considérant indépendamment et avec le même poids les trois variables hauteur d'eau, vitesse du courant et substrat (aucune pondération n'est introduite dans la multiplication des coefficients), et ceci quelle que soit l'activité. Cependant, des études écoéthologiques telles que celle de BALDES et VINCENT (1969) en chenal expérimental, montrent que les truites adultes (Salmo trutta fario) se répartissent préférentiellement en fonction de certaines associations de variables dans lesquelles la hauteur d'eau joue un rôle prépondérant.

D'autre part, les courbes de préférence sont inféodées à des valeurs absolues des variables, alors que les préférences des individus sont soumises à des variations liées à la disponibilité en habitats (GROSSMAN et al., 1987). Le déplacement des pics de préférence observé entre les courbes de préférence présentées ici et celles établies par BARAS (1992) illustre ce phénomène et explique en partie la perte de qualité de prédiction lors de l'utilisation de modèles généraux.

A l'inverse, l'approche multivariée met tout d'abord en avant les variables prépondérantes dans le choix de l'habitat (la vitesse du courant puis la hauteur d'eau dans notre cas) en modifiant leur importance relative sur chacun des axes de l'analyse. De plus la prédiction n'est plus réalisée à partir d'une valeur absolue d'une variable mais à partir de la combinaison des trois variables, ce qui correspondrait plus, selon BALDES et VINCENT (1969), au mode de sélection utilisé par les poissons. L'approche multivariée permet d'ores et déjà de répondre à certaines critiques (notamment de ne plus imposer l'hypothèse d'indépendance des variables, de prendre en compte simultanément les dimensions biologiques et abiotiques). Elle offre des perspectives d'amélioration des plus intéressantes pour aboutir à des solutions plus fiables, mimant au plus près les phénomènes importants du fonctionnement interne des cours d'eau. En outre, les analyses multivariées permettent l'adjonction, sans aucune restriction, de nouvelles variables importantes dans la sélection de l'habitat par les poissons (abris, végétation, par exemple). 
Par ailleurs, en réponse à des enjeux plus complexes de gestion des hydrosystèmes, comme la conservation de la biodiversité, les méthodes d'évaluation multivariées peuvent offrir une nouvelle échelle de résolution, évitant le choix d'une espèce cible, en estimant simultanément une capacité potentielle de toutes les espèces constituant le peuplement.

\section{REMERCIEMENTS}

Ces recherches sont inscrites dans le programme PIR POISSON “Modèles d'habitat" du CNRS (SOUCHON et GAUDIN, 1993) et ont également bénéficié du soutien financier du Ministère de l'Environnement, Direction de l'Eau (contrat 91289) et DRAEI (contrat 90207).

\section{BIBLIOGRAPHIE}

ALLEE W.C., EMERSON H.E., PARK O., PARK T., SCHMIDT K.P., 1949. Principles of animal ecology. Saunders W.B., Philadelphia, Pennsylvania, USA.

BALDES R.J., VINCENT R.E., .1969. Physical parameters of microhabitats occupied by brown trout in an experimental flume. Trans. Am. Fish. Soc., 98, 230-238.

BARAS E., CHERRY B., 1990. Seasonal activities of female barbel Barbus barbus (L.) in the river Ourthe as revealed by radio tracking. Aquat. Liv. Resour., 3 (4), 283-294.

BARAS E., 1992. Contribution à l'étude des stratégies d'occupation du temps et de l'espace chez un poisson téléostéen dulcicole, le barbeau fluviatile, Barbus barbus (L.). Etude par radiopistage, pêche à l'électricité et observation directe. Thèse de Doctorat, Univ. Liège.

BARAS E., 1993. Etude par biotélémétrie de l'utilisation de l'espace, chez le barbeau fluviatile, Barbus barbus (L.). Caractérisation et implications des patrons saisonniers de mobilité. In PONCIN P., BERREBI P., PHILIPPART J.C., et RUWEIT J.C. (eds), Actes de la Table Ronde Internationale Barbus II. Cahiers d'Ethologie, 13 (2), 135-138.

BAUME J.P., POIRSON M., 1984. Modélisation numérique d'un écoulement permanent dans un réseau hydraulique maillé à surface libre, en régime fluvial. La Houille Blanche, 1 (2), 95-100.

BOVEE K.D., MILHOUS R.T., 1978. Hydraulic simulation in instream flow studies : theory and techniques. Paper $n^{\circ} 5$ Instream flow information. Western Energy and Land Use. Ft Collins, Colorado. FWS/OBS 78/33, 143p.

BOVEE K.D., 1982. A guide to stream habitat analysis using the Instream Flow Incremental Methodology. Paper $n^{\circ} 12$ Instream flow information. Western Energy and Land Use. Ft Collins, Colorado. FWS/OBS 82/26, 248p.

CAZES P., 1980. L'analyse de certains tableaux rectangulaires décomposés en blocs : généralisation des propriétés rencontrées dans l'étude des correspondances multiples. Cah. Ana. Données, 5 (2), 145-161; 5 (3), 387-403.

CHESSEL D., DOLEDEC S., 1991. ADE version 3.1. Hypercard stacks and Quickbasic microsoft programme library for the analyse of environmental data. URA CNRS 1451, Univ. Lyon I, 43 Bd 11 Novembre 1918, 69622 Villeurbanne Cedex, France.

DOLEDEC S., CHESSEL D., 1994. Co-inertia analysis : an alternative method for studying species-environment relationships. Freshw. Biol., 31, 277-294.

GINOT V., SOUCHON Y., 1992. EVHA : un système informatique pour évaluer l'habitat du poisson. Actes du Colloque ADEME «insertion des petites centrales hydroélectriques dans l'environnement", 158-166.

GORMAN O. T., KARR J.R., 1978. Habitat structure and stream fish communities. Ecol., 59 (3), 507-515.

GROSSMAN G.D., DE SOSTOA A., FREEMAN M.C., LOBON-CERVIA J., 1987. Microhabitat use in a Mediterranean riverine fish assemblage. Fishes of the lower Matarrana. Oecologia, 73, 490-500. 
HANSON D.F., 1988. Investigations into the use of bivariate habitat suitability functions in application of the Phabsim model. In BOVEE K.D. and ZUBOY J.R. (eds), Proceedings of a workshop on the development and evaluation of habitat suitability criteria. U.S. Wildl. Serv. Biol. Rep., 88 (11), 407p.

HEGGENES J., BRABANT A., SALVEIT S.J., 1990. A comparison of three different methods for studies of stream habitat use by brown Trout and Atlantic salmon. Trans. Am. Fish. Soc., 119, 101-111.

HUET M., 1949. Aperçu des relations entre la pente et les populations piscicoles des eaux courantes. Rev. Suisse d'Hydrobiol., 11, 332-351.

HUGHES R.M., NOSS R.F., 1992. Biological diversity and biological integrity : current concerns for lakes and streams. Fisheries, 17 (3), 11-19.

LEONARD P.M., ORTH D.J., GOUDREAU C.J., 1986. Development of a method for recommending instream flows for fisheries in the upper James river, Virginia. Bulletin of Virginia Water Resources Research Center $n^{\circ} 152,122 \mathrm{p}$.

MALAVOI J.R., 1989. Typologie des faciès d'écoulement ou unités morphodynamiques des cours d'eau à haute énergie. Bull. Fr. Pêche Piscic., 315, 189-210.

MALAVOI J.R., SOUCHON Y., 1989. Méthodologie de description et de quantification des variables morphodynamiques d'un cours d'eau à fond caillouteux. Rev. Géo. Lyon, 64 (4), 252-259.

MATHUR D., BASON W.H., PURDY E.J., SILVER C.A., 1985. A critique of the Instream Flow Incremental Methodology. Can. J. Fish. Aquat. Sci., 42, 825-831.

MERCIER P., CHESSEL D., DOLEDEC S., 1992. Complete correspondence analysis of an ecological profile table : a central ordination method. Acta Oecol. Gener., 13(1), $25-44$.

MOYLE P.B., VONDRACEK B., 1985. Persistence and structure of the fish assemblage in a small california stream. Ecol., 66, 1-13.

NELVA A., PERSAT H., CHESSEL D., 1979. Une nouvelle méthode d'étude des peuplements ichtyologiques dans les grands cours d'eau par échantillonnage ponctuel d'abondance. C.R. Acad. Sc., 289(D), 1295-1298.

ORTH D.J., MAUGHAN O.E., 1982. Evaluation of the incremental methodology for recommending instream flow for fishes. Trans. Am. Fish. Soc., vol. 3 (4), 413-445.

ORTH D.J., 1987. Ecological considerations in the development and application of instream flow-habitat models. Reg. Riv. : Res. and Manage., 1, 171-181.

OSBORNE L.L., BAYLEY P.B., HIGLER L.W., 1993. Lowland stream restoration : theory and practice. Freshw. Biol., 29 (2), 342p.

PELZ G.R., KASTLE A., 1989. Ortsbewegungen der barbe Barbus barbus (L.) radiotelemetrische standortbestimmungen in der Nidda. Fischökologie, 1, 15-28.

POUILLY M., SOUCHON Y., 1989. Schéma départemental de vocation piscicole du fleuve Rhône. Simulation des capacités d'habitat potentiel des poissons. CEMAGREF Lyon, 56p.

POUILLY M., 1994. Relations entre l'habitat physique et les poissons des zones à cyprinidés rhéophiles dans trois cours d'eau du bassin rhodanien : vers une simulation de la capacité d'accueil pour les peuplements. Thèse Univ. Lyon I / CEMAGREF Lyon, 260p.

SCHLOSSER I.J., 1982. Fish community structure and function along two habitat gradients in a headwater stream. Ecological monographs, 52 (4), 395-414.

SHIRVELL C.S., 1989. Ability of Phabsim to predict chinook salmon spawning habitat. Reg. Riv. : Res. and Manage., 3, 277-289.

SOUCHON Y., TROCHERIE F., FRAGNOUD E., LACOMBE C., 1989. Les modèles numériques des microhabitats des poissons : application et nouveaux développements. Rev. Sci. Eau, 2, 807-830. 
SOUCHON Y., GAUDIN P., 1993. PIR “Facteurs de l'environnement, utilisation de l'espace et structuration spatio-temporelle des peuplements piscicoles". Modélisation de l'utilisation spatio-temporelle de l'habitat par les poissons : exemples de la truite, de l'ombre commun et du barbeau fluviatile. CNRS - URA 1451 "Ecologie des Eaux douces et des Grands Fleuves", CEMAGREF BEA/LHQ, INRA Département d'Hydrobiologie. Rapport d'étape $2^{\mathrm{e}}$ année.

THOMAS J.A., BOVEE K.D., 1993. Application and testing of a procedure to evaluate transferability of habitat suitability criteria. Reg. Riv. : Res. and Manage., 8 (3), 285-294.

TROCHERIE F., 1987. Mise en application de l'article 410 du code rural. Elément d'une démarche de détermination des débits réservés. CEMAGREF Lyon, Ministère de l'environnement, Direction de la Protection de la Nature. Rapport final convention DPN, 85-15.

VOOS K.A., LIFTON W.S., 1988. Development of a bivariate depth and velocity suitability function for dolly varden (Salvelinus malma) Juveniles. In BOVEE K.D. et ZUBOY J.R. (eds), Proceedings of a workshop on the development and evaluation of habitat suitability criteria. U.S. Wild. Serv. Biol. Rep., 88 (11), 407p. 